\title{
Comparação da dor e qualidade de vida entre indivíduos com e sem neuropatia diabética
}

Comparison of pain and quality of life between individuals with and without diabetic neuropathy Comparación del dolor y la calidad de vida entre individuos con y sin neuropatía diabética

\section{Ana Catarina Guimarães Silva', Marina Morato StivalI", Silvana Schwerz Funghetto ${ }^{I I I}$, Cris Renata Grou Volpe ${ }^{\mathrm{IV}}$, Mani Indiana Funez ${ }^{\mathrm{v}}$, Luciano Ramos de Lima ${ }^{\mathrm{VI}}$}

\begin{abstract}
Resumo: Objetivo: comparar a dor e a qualidade de vida de indivíduos com e sem neuropatia diabética. Método: estudo transversal realizado com 251 participantes com diabetes mellitus tipo 2. Utilizou-se a escala Leeds Assessment of Neuropathic Symptoms and Signs (LANSS) para avaliar neuropatia diabética, características da dor, perda de sensibilidade protetora (PSP) e avaliar a qualidade de vida pelo Short-Form 6 Dimensions-Brasil/SF-6D. Realizou-se análise estatística descritiva. Resultados: 16,3\% apresentaram neuropatia, 97,6\% queixaram-se de dor, sendo a maioria crônica e nos pés ou panturrilhas. $51,2 \%$ dos neuropáticos tiveram PSP no teste do monofilamento $(\mathrm{p}=0,001)$. Os descritores de dor mais referidos pelos neuropáticos: queimação $(\mathrm{p}=0,004)$, formigamento $(\mathrm{p}=0,002) \mathrm{e}$ alfinetada e/ou agulhada $(\mathrm{p}=0,003)$ e os domínios de qualidade de vida afetados foram: dor, saúde mental e vitalidade. Conclusão: aqueles com neuropatia têm maior intensidade de dor, acordam à noite e apresentam alteração na sensibilidade dos pés, que pode ser rastreada na atenção primária.
\end{abstract}

Descritores: Enfermagem; Diabetes Mellitus; Neuropatias Diabéticas; Qualidade de Vida; Atenção Primária à Saúde

Abstract: Objective: to compare pain and quality of life in individuals with and without diabetic neuropathy. Method: a cross-sectional study with 251 participants with type 2 diabetes mellitus. The Leeds Assessment of Neuropathic Symptoms and Signs (LANSS) scale was used to assess diabetic neuropathy, pain characteristics, loss

\footnotetext{
${ }^{\text {I }}$ Enfermeira, Graduada em enfermagem pela Universidade de Brasília-Campus Ceilândia/UNB/FCE, Bolsista de Iniciação científica. Brasília, Distrito Federal, Brasil. E-mail: anacatarina.enf@gmail.com, Orcid: https://orcid.org/0000-0002-3045-528x

II Enfermeira, Doutora, UNB/FCE, Professora adjunta do curso de enfermagem. Brasília, Distrito Federal, Brasil. E-mail: marinamorato@unb.br, Orcid: https://orcid.org/0000-0001-6830-4914

III Enfermeira, Doutora, UNB/FCE, Professora adjunta do curso de enfermagem. Brasília, Distrito Federal, Brasil. E-mail: silvana.funghetto@gmail.com, Orcid: https://orcid.org/0000-0002-9332-9029

IV Enfermeira, Doutora, UNB/FCE, Professora adjunta do curso de enfermagem. Brasília, Distrito Federal, Brasil. E-mail: crgrou@unb.br, Orcid: https://orcid.org/0000-0002-3901-0914

V Enfermeira, Doutora, UNB/FCE, Professora associada do curso de enfermagem. Brasília, Distrito Federal, Brasil. E-mail: mani@unb.br. Orcid: https://orcid.org/0000-0002-4315-7185

VI Enfermeiro, Doutor, UNB/FCE. Professor adjunto do curso de enfermagem. Brasília, Distrito Federal, Brasil. E-mail: ramosll@unb.br, Orcid: https://orcid.org/0000-0002-2709-6335
} 
of protective sensitivity (LPS) and to assess the quality of life by Short-Form 6 Dimensions-Brasil/SF-6D. Descriptive statistical analysis was performed. Results: among the participants, $16.3 \%$ had neuropathy, $97.6 \%$ complained of pain, most of them chronic and in the feet or calves. Also, $51.2 \%$ of neuropathic patients had LPS in the monofilament test $(\mathrm{p}=0.001)$. The pain descriptors most frequently reported by neuropathic patients were: burning $(\mathrm{p}=0.004)$, tingling $(\mathrm{p}=0.002)$, and pinprick and/or needling $(\mathrm{p}=0.003)$. The affected quality of life domains were: pain, mental health, and vitality. Conclusion: those with neuropathy have greater pain intensity, wake up at night, and have altered foot sensitivity, which can be tracked in primary care.

Descriptors: Nursing; Diabetes Mellitus; Diabetic Neuropathies; Quality of Life; Primary Health Care

Resumen: Objetivo: comparar el dolor y la calidad de vida en individuos con y sin neuropatía diabética. Método: estudio transversal con 251 participantes con diabetes mellitus tipo 2. Se utilizó la escala Leeds Assessment of Neuropathic Symptoms and Signs (LANSS) para evaluar la neuropatía diabética, las características del dolor, la pérdida de sensibilidad protectora (PSP) y para evaluar la calidad de vida por Short-Form 6 Dimensiones-Brasil/SF6D. Se realizó análisis estadístico descriptivo. Resultados: el 16,3\% presentaba neuropatía, el 97,6\% se quejaba de dolor, la mayoría crónico y en pies o pantorrillas. El 51,2\% de los pacientes neuropáticos tenían PSP en la prueba de monofilamento $(\mathrm{p}=0,001)$. Los descriptores de dolor informados con mayor frecuencia por los pacientes neuropáticos fueron: ardor $(\mathrm{p}=0,004)$, hormigueo $(\mathrm{p}=0,002)$ y pinchazo y/o punción $(\mathrm{p}=0,003)$ y los dominios de calidad de vida afectada fueron: dolor, salud mental y vitalidad. Conclusión: las personas con neuropatía tienen mayor intensidad de dolor, se despiertan por la noche y tienen alteración de la sensibilidad de los pies, lo que se puede rastrear en atención primaria.

Descriptores: Enfermería; Diabetes Mellitus; Neuropatías Diabéticas; Calidad de Vida; Atención Primaria de Salud

\section{Introdução}

De acordo com a Sociedade Brasileira de Diabetes e a International Diabetes Federation, no mundo, 1 a cada 11 adultos tem diabetes mellitus (DM), o que representa aproximadamente 424,9 milhões de pessoas. Também é previsto que em 2045 cerca de 628,6 milhões de pessoas terão DM no mundo. Entretanto, 50\% desses indivíduos ainda não foram diagnosticados. ${ }^{1-2}$ Nos países em desenvolvimento, o número de casos de DM tem aumentado de forma crescente. O Brasil possui a estimativa de 14,3 milhões de indivíduos com DM, cerca de $9,4 \%$ da população. ${ }^{3-4}$ Após o diagnóstico, o controle adequado da doença é fundamental, pois objetiva prevenir complicações advindas do DM, que a longo prazo podem ter complicações como presença de dor crônica e, consequentemente, afetar a qualidade de vida (QV).2-3 As complicações do DM têm alta prevalência e podem ser classificadas em microvasculares (nefropatia, retinopatia e neuropatia) e macrovasculares (doença vascular periférica, doença arterial coronariana e 
acidentes vasculares encefálicos). ${ }^{2,5}$ Neste sentido, destaca-se a neuropatia diabética (ND), uma complicação que pode estar presente até mesmo na fase que antecede o próprio DM (prédiabetes) ${ }^{6}$ e principalmente no diabetes mellitus tipo 2 (DM2), na presença de hiperglicemia positivamente relacionada com complicações microvasculares. ${ }^{1-2,5}$ A ND acomete cerca de $50 \%$ dos pacientes e geralmente ocorre após dez anos do diagnóstico de DM2, que pode ser considerada dolorosa ou não dolorosa..$^{6-7}$

A ND tem como principais manifestações clínicas a parestesia ou queimação de membros inferiores (MMII), formigamento, pontadas, choques, agulhadas em pernas e pés, desconforto ou dor ao toque leve e/ou mínimo estímulo e diminuição ou perda de sensibilidade tátil, térmica ou dolorosa. Os sinais e sintomas acometem progressivamente os MMII e podem afetar simetricamente os membros superiores., ${ }^{2,5}$ Assim, a dor é considerada uma forma de sinalizar complicações neurológicas de MMII advindas do DM2. ${ }^{2}$

Em geral, à medida que a doença progride, esses pacientes podem apresentar dor e a QV alterada, já que a patologia está associada a limitações e a elevado grau de incapacidade, gerando afastamento do trabalho, diminuição da produtividade e dificuldade de realização das atividades da vida diária..$^{8-9} \mathrm{~A}$ dor neuropática esteve presente em cerca de $18,0 \%$ dos participantes de um estudo realizado com indivíduos com DM2 na Espanha. ${ }^{5}$ No Brasil varia entre $19,1 \%$ e $58 \%$ dependendo do local e do método de diagnóstico dos pacientes com ND.9-10 Além disso, a dor neuropática pode tornar-se crônica e incapacitante e seus sintomas desencadeados muitas vezes não respondem à terapêutica, o que afeta ainda mais a QV do paciente, podendo suscitar isolamento social e depressão., ${ }^{5,11}$

Por essa razão, estudos revelam que, entre pacientes com DM2 que sofrem com complicações crônicas, há demonstração de QV inferior aos que não tem DM2. ${ }^{12-13}$ As complicações microvasculares, especificamente, apresentam maior prevalência e promovem altas taxas de internações, amputações não traumáticas e incapacidades.,10 Pesquisadores 
Comparação da dor e qualidade de vida entre indivíduos com e sem neuropatia diabética | 4

pontuam que as complicações microvasculares estão associadas significativamente a uma pior QV quando comparadas às complicações macrovasculares..,11-12

Assim, a QV e a dor têm sido investigadas em pacientes com DM2 com utilização de variados instrumentos. ${ }^{11-15}$ Contudo a investigação da dor pela escala de Leeds Assessment of Neuropathic Symptoms and Signs - LANSS tem sido pouco utilizada no Brasil em relação aos estudos internacionais. ${ }^{16-20}$ Dessa forma, reconhecer a dor com características de ND é importante para identificar as complicações do DM, além do fato de utilizar instrumentos validados que ajudam a construir o conhecimento de enfermagem. Ressalta-se que os pacientes buscam a atenção básica como porta de entrada para o diagnóstico e tratamento das complicações advindas do DM2. Diante disso, a dor da ND é considerada um grave problema.

Após o exposto, este estudo teve como objetivo comparar a dor e a QV de indivíduos com e sem ND.

\section{Método}

Trata-se de um estudo transversal. A pesquisa foi realizada em duas Unidades Básicas de Saúde (UBS) da regional de saúde oeste do Distrito Federal/DF, Brasil. A população desta pesquisa foi composta por pacientes com DM do tipo 2 cadastrados nas referidas UBS.

O cálculo amostral considerou um erro amostral de 5\% e intervalo de confiança de 95\%, que somou um número final de 251 participantes. A seleção amostral ocorreu de forma aleatória por meio de sorteio mediante o número de cadastro na UBS. A coleta de dados ocorreu entre abril e julho de 2017 .

Primeiramente, foi agendado com os pacientes sorteados, por contato telefônico, um dia específico para comparecimento na UBS, onde, em uma sala privada, foram realizadas todas as avaliações. Foram incluídos na amostra os indivíduos que atenderam aos seguintes critérios de inclusão: ter o diagnóstico de DM do tipo $2 \geq 06$ meses, idade $\geq 18$ anos, ser cadastrado e realizar 
5 | Silva ACG, Stival MM, Funghetto SS, Volpe CRG, Funez MI, LimaVI LR

acompanhamento na UBS, ter capacidade de compreender, verbalizar e responder aos questionários. Foram excluídos do estudo gestantes, pessoas com diagnóstico médico de doenças mentais e neoplasias em tratamento, e aqueles sem possibilidade de avaliação dos pés. Foram selecionados 256 pacientes em um primeiro momento, contudo cinco participantes foram excluídos por não ser possível realizar a avaliação dos pés devido à maceração interdigital (três), micose (um) e erisipela (um) dos participantes. A avaliação dos pacientes ocorreu por meio de aplicação de instrumentos estruturados que caracterizavam os perfis demográfico, socioeconômico e clínico, a avaliação da dor com características de neuropatia e da QV.

Para avaliação da dor com características de ND, foi considerada a presença de dor nas panturrilhas e/ou nós pés, que apresentou escore $\geq 12$ pontos na Escala LANSS. A escala explora aspectos qualitativos e sensitivos da dor com pontuação variando entre 0 e 24 pontos, de modo que o escore $\geq 12$ pontos indica que provavelmente mecanismos neuropáticos estejam contribuindo para a dor. ${ }^{14}$ Também foi pesquisada a Perda de Sensibilidade Protetora (PSP) por meio do monofilamento de Semmes-Weinstein (10g). Este foi aplicado na falange distal do hálux em região plantar; cabeça do primeiro, terceiro e quinto metatarso plantar. ${ }^{2,4}$

Outra avaliação foi a mensuração da dor, caracterizando a prevalência, localização, duração, intensidade e qualidade. A prevalência e a duração foram investigadas pela classificação em dor crônica (tempo de dor maior que 6 meses). A intensidade foi mensurada pela Escala Visual Analógica (EVA) de 0 a 10 pontos. A intensidade de dor nos pés e panturrilhas avaliada pela EVA foi categorizada em ausente (zero), leve (1 a 3 pontos), moderada (4 a 6 pontos) e intensa (7 a 10 pontos). ${ }^{4}$ A qualidade da dor foi avaliada pelo instrumento McGill, por meio do uso de descritores da dor, podendo ser referida como: queimação, dormência, formigamento, fadiga, câimbras, prurido, alfinetada, agulhada e outros. ${ }^{15}$

Por fim, a última avaliação foi a investigação da QV, para a qual se utilizou o Instrumento Short-Form 6 Dimensions-Brasil-SF-6D (SF-6D). Traduzido e validado para o Brasil, o 
Comparação da dor e qualidade de vida entre indivíduos com e sem neuropatia diabética |6

instrumento SF-6D foi elaborado do questionário SF-36, sendo capaz de avaliar a QV nas seguintes dimensões: capacidade funcional, limitação global, aspectos sociais, dor, saúde mental e vitalidade. O escore desse instrumento representa a preferência do indivíduo por estados de saúde. A pontuação varia de 0 a 1 , em que 0 equivale ao pior estado de saúde e 1 corresponde ao melhor estado de saúde. ${ }^{16}$

Foi construído um banco de dados no Software Package for the Social Sciences (SPSS ${ }^{\circledR}$ ) versão 21.0. A análise estatística descritiva foi realizada por meio do cálculo de frequências absolutas, relativas e medidas de dispersão (média, desvio padrão, mínima e máxima). O teste Qui-quadrado foi usado para comparação das proporções entre os grupos. Após as variáveis numéricas apresentarem distribuição normal no teste de Kolmogorov-Smirnov, compararam-se as médias entre os grupos por meio do teste t-student. Considerou-se $\mathrm{p}<0,05$.

A assinatura por todos os participantes do Termo de Consentimento Livre e Esclarecido foi garantida, seguindo os preceitos éticos da resolução 466/2012. Este estudo foi aprovado pelo Comitê de Ética em Pesquisa da Fundação de Ensino e Pesquisa em Ciências da Saúde da Secretária de Saúde do Distrito Federal sob o parecer n ${ }^{\circ}$ 1.355.211.

\section{Resultados}

A caracterização dos participantes com DM2 evidenciou uma idade média de 58,9ะ10,01 anos (Mín.=30 e Máx.=85 anos). Prevaleceram mulheres (74,5\%), participantes com idade entre 51 e 60 anos $(37,8 \%)$, de cor parda $(49,8 \%)$, natural da região Nordeste $(56,2 \%)$, casados $(49,0 \%)$, com ensino fundamental $(65,3 \%)$, ativos $(35,1 \%)$, com renda mensal menor ou igual a 1 salário mínimo $(45,8 \%)$, com tempo de doença menor que 10 anos (68,5\%) e com hipertensão arterial (79,7\%). De acordo com os escores de avaliação pela escala de LANSS, 16,3\% dos pacientes apresentaram neuropatia (Tabela 1). 
Tabela 1 - Comparação do perfil clínico e sociodemográfico de indivíduos com DM tipo 2 com e sem neuropatia ( $\mathrm{n}=251)$, Região Oeste, Brasília-DF, 2017.

\begin{tabular}{|c|c|c|c|c|c|c|c|c|}
\hline & & \multicolumn{6}{|c|}{ Escala de dor LANSS } & \multirow{3}{*}{$\mathrm{p}$} \\
\hline & & \multicolumn{2}{|c|}{ Total $n=251$} & \multicolumn{2}{|c|}{ Com neuropatia $n=41$} & \multicolumn{2}{|c|}{ Sem neuropatia $n=210$} & \\
\hline & & $\mathrm{n}$ & $\%$ & $\mathrm{n}$ & $\%$ & $\mathrm{n}$ & $\%$ & \\
\hline \multirow[t]{4}{*}{ Sexo } & Masculino & 64 & 25,5 & 8 & 19,5 & 56 & 26,7 & 0,336 \\
\hline & Feminino & 187 & 74,5 & 33 & 80,5 & 154 & 73,3 & \\
\hline & 30 e 40 anos & 7 & 2,8 & 3 & 7,3 & 4 & 1,9 & \\
\hline & 41 e 50 anos & 47 & 18,7 & 10 & 24,4 & 37 & 17,6 & \\
\hline \multirow[t]{4}{*}{ Idade } & 51 e 60 anos & 95 & 37,8 & 14 & 34,1 & 81 & 38,6 & 0,753 \\
\hline & 61 e 70 anos & 67 & 26,7 & 9 & 22 & 58 & 27,6 & \\
\hline & $\geq 71$ anos & 35 & 13,9 & 5 & 12,2 & 30 & 14,3 & \\
\hline & Branco & 70 & 27,9 & 8 & 19,5 & 62 & 29,5 & \\
\hline \multirow{4}{*}{ Cor } & Pardo & 125 & 49,8 & 20 & 48,8 & 105 & 50 & \\
\hline & Negro & 47 & 18,7 & 12 & 29,3 & 35 & 16,7 & 0,228 \\
\hline & Outros & 9 & 3,6 & 1 & 2,4 & 8 & 3,8 & \\
\hline & Norte & 8 & 3,2 & 3 & 7,3 & 5 & 2,4 & \\
\hline \multirow{4}{*}{ Naturalidade } & Nordeste & 141 & 56,2 & 19 & 46,3 & 122 & 58,1 & \\
\hline & Centro-Oeste & 56 & 22,3 & 10 & 24,4 & 46 & 21,9 & 0,343 \\
\hline & Sudeste & 44 & 17,5 & 9 & 22 & 35 & 16,7 & \\
\hline & Solteiro & 51 & 20,3 & 8 & 19,5 & 43 & 20,5 & \\
\hline \multirow{4}{*}{ Estado Civil } & Casado & 123 & 49,0 & 19 & 46,3 & 104 & 49,5 & \\
\hline & Divorciado & 37 & 14,7 & 11 & 26,8 & 26 & 12,4 & 0,065 \\
\hline & Viúvo & 40 & 15,9 & 3 & 7,3 & 37 & 17,6 & \\
\hline & Analfabeto & 13 & 5,2 & 3 & 7,3 & 10 & 4,8 & \\
\hline \multirow{3}{*}{ Escolaridade } & Fundamental & 164 & 65,3 & 24 & 58,5 & 140 & 66,7 & \\
\hline & Médio & 67 & 26,7 & 12 & 29,3 & 55 & 26,2 & 0,644 \\
\hline & Superior & 7 & 2,8 & 2 & 4,9 & 5 & 2,4 & \\
\hline \multirow{6}{*}{$\begin{array}{l}\text { Situação de } \\
\text { atividade }\end{array}$} & Ativo & 88 & 35,1 & 16 & 39 & 72 & 34,3 & \\
\hline & Aposentado & 60 & 23,9 & 10 & 24,4 & 50 & 23,8 & \\
\hline & Afastado & 8 & 3,2 & 1 & 2,4 & 7 & 3,3 & 0,974 \\
\hline & Desempregado & 81 & 32,3 & 12 & 29,3 & 69 & 32,9 & \\
\hline & Sem resposta & 14 & 5,6 & 2 & 4,9 & 12 & 5,7 & \\
\hline & $\leq 1 \mathrm{SM}^{*}$ & 115 & 45,8 & 20 & 48,8 & 95 & 45,2 & \\
\hline \multirow{3}{*}{ Salário mínimo } & 2 a $3 S^{*}$ & 95 & 37,8 & 18 & 43,9 & 77 & 36,7 & \\
\hline & 4 a $5 S^{*}$ & 23 & 9,2 & 1 & 2,4 & 22 & 10,5 & 0,341 \\
\hline & 6 ou mais $\mathrm{SM}^{*}$ & 18 & 7,2 & 2 & 4,9 & 16 & 7,6 & \\
\hline Tempo de DM & $<10$ anos & 172 & 68,5 & 31 & 75,6 & 141 & 67,1 & \\
\hline \multirow[t]{3}{*}{+} & $>10$ anos & 66 & 26,3 & 8 & 19,5 & 58 & 27,6 & 0,542 \\
\hline & Não sabe & 13 & 5,2 & 2 & 4,9 & 11 & 5,2 & \\
\hline & $\mathrm{HAS}^{*}$ & 200 & 79,7 & 35 & 85,4 & 165 & 78,6 & \\
\hline \multirow[t]{2}{*}{ Comorbidade } & Outras & 3 & 1,2 & 1 & 2,4 & 2 & 1 & 0,356 \\
\hline & Nenhuma & 48 & 19,1 & 5 & 12,2 & 43 & 20,5 & \\
\hline
\end{tabular}

*Salário mínimo: SM (R\$937,00) em 2017; ${ }^{+} \mathrm{DM}$ : diabetes mellitus; ${ }^{*} \mathrm{HAS}$ : hipertensão arterial sistêmica. 
Comparação da dor e qualidade de vida entre indivíduos com e sem neuropatia diabética |8

A prevalência de dor foi de $97,6 \%$, ressaltando-se que todos os pacientes com neuropatia referiram dor. Quanto à localização da principal dor, 35,1\% referiram dor em MMII e 83,3\% alegaram dor em pés ou panturrilhas, de maneira que $90,2 \%$ dos neuropáticos queixavam-se desse acometimento. A maioria dos participantes apresentou dor crônica. Um maior número de pacientes com neuropatia referiu acordar à noite frequentemente e continuamente devido à dor quando comparado ao grupo sem neuropatia $(\mathrm{p}=0,000)$. A dor foi descrita como intensa pela maioria dos participantes (Tabela 2).

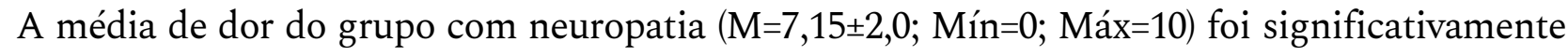
maior do que a do grupo sem neuropatia $(M=6,4 \pm 2,3$; Mín=0; Máx=10) (p=0,038).

Tabela 2 - Comparação da avaliação da dor quanto à prevalência, à localização, à duração, à intensidade e à qualidade em indivíduos com DM tipo 2 com e sem neuropatia ( $\mathrm{n}=251)$ Região Oeste, Brasília-DF, 2017.

\begin{tabular}{|c|c|c|c|c|c|c|c|c|}
\hline & & \multicolumn{7}{|c|}{ Escala de dor LANSS } \\
\hline & & \multicolumn{2}{|c|}{ Total $n=251$} & \multicolumn{2}{|c|}{ Com neuropatia $n=41$} & \multicolumn{2}{|c|}{ Sem neuropatia $n=210$} & \multirow[t]{2}{*}{$\mathrm{p}$} \\
\hline & & $\mathrm{n}$ & $\%$ & $\mathrm{n}$ & $\%$ & $\mathrm{n}$ & $\%$ & \\
\hline \multirow{5}{*}{$\begin{array}{l}\text { Presença de } \\
\text { dor }\end{array}$} & $\operatorname{Sim}$ & 245 & 97,6 & 41 & 100 & 204 & 97,1 & 0,273 \\
\hline & Não & 6 & 2,4 & -- & -- & 6 & 2,9 & \\
\hline & ${ }^{*} \mathrm{MMII}$ & 88 & 35,1 & 15 & 36,6 & 73 & 34,8 & \\
\hline & ${ }^{+} \mathrm{MMSS}$ & 23 & 9,2 & 3 & 7,3 & 20 & 9,5 & \\
\hline & Região dorsal & 53 & 21,1 & 9 & 22 & 44 & 21 & \\
\hline \multirow{4}{*}{$\begin{array}{l}\text { Localização } \\
\text { principal }\end{array}$} & Região ventral & 8 & 3,2 & 1 & 2,4 & 7 & 3,3 & 0,509 \\
\hline & Região cefálica & 13 & 5,2 & -- & -- & 13 & 6,2 & \\
\hline & $\begin{array}{l}\text { Mais de uma } \\
\text { localização }\end{array}$ & 60 & 23,9 & 13 & 31,7 & 47 & 22,4 & \\
\hline & Ausência de dor & 6 & 2,4 & -- & -- & 6 & 2,9 & \\
\hline \multirow{3}{*}{$\begin{array}{l}\text { Tempo de } \\
\text { dor }\end{array}$} & $<6$ de meses & 32 & 12,7 & 3 & 7,3 & 29 & 13,8 & \\
\hline & $>6$ de meses & 213 & 84,9 & 38 & 92,7 & 175 & 83,3 & 0,265 \\
\hline & Ausência de dor & 6 & 2,4 & -- & -- & 6 & 2,9 & \\
\hline \multirow{2}{*}{$\begin{array}{l}\text { Dor nos pés } \\
\text { ou } \\
\text { panturrilha }\end{array}$} & Sim & 209 & 83,3 & 37 & 90,2 & 172 & 81,9 & 0,191 \\
\hline & Não & 42 & 16,7 & 4 & 9,8 & 38 & 18,1 & \\
\hline \multirow{3}{*}{$\begin{array}{l}\text { Desperta } \\
\text { devido à dor }\end{array}$} & Nunca & 116 & 46,2 & 6 & 14,6 & 110 & 52,4 & \\
\hline & Às vezes & 70 & 27,9 & 15 & 36,6 & 55 & 26,2 & \\
\hline & Frequentemente & 43 & 17,1 & 15 & 36,6 & 28 & 13,3 & 0,000 \\
\hline
\end{tabular}


9 | Silva ACG, Stival MM, Funghetto SS, Volpe CRG, Funez MI, LimaVI LR

\begin{tabular}{llccccccc} 
& Continuamente & 16 & 6,4 & 5 & 12,2 & 11 & 5,2 & \\
& Ausência de dor & 6 & 2,4 & -- & -- & 6 & 2,9 & \\
& Ausente & 43 & 16 & 4 & 9,8 & 38 & 18,1 & \\
${ }^{*}$ EVA & 9 & 4,3 & -- & -- & 9 & 4,3 & \\
& Leve & 85 & 33,9 & 12 & 29,3 & 73 & 34,8 & 0,118 \\
& Moderada & 115 & 45,8 & 25 & 61 & 90 & 42,9 & \\
\hline
\end{tabular}

*MMII: membros inferiores; ${ }^{+}$MMSS: membros superiores; ${ }^{*}$ EVA: Escala Visual Analógica.

Na avaliação dos pés pela PSP, observou-se que a maioria dos indivíduos do grupo com neuropatia apresentou alterações no teste do monofilamento de $10 \mathrm{~g}(\mathrm{p}=0,001)$. A respeito da qualidade da dor, os principais descritores mencionados foram: fadiga, queimação, câimbra e alfinetada e/ou agulhada. Entre aqueles com neuropatia, os mais referidos em comparação aos sem neuropatia foram: queimação $(\mathrm{p}=0,004)$, formigamento $(\mathrm{p}=0,002)$ e alfinetada e/ou agulhada $(\mathrm{p}=0,003)$ (Tabela 3$)$.

Tabela 3 - Comparação da avaliação de dor pela Escala de McGill, exame de perda da sensibilidade protetora plantar (PSP) e descritores de dor neuropática de indivíduos com DM tipo 2 com e sem neuropatia (n=251), Região Oeste, Brasília-DF, 2017.

\begin{tabular}{lccccccc}
\hline & \multicolumn{7}{c}{ Escala de dor LANSS } \\
\cline { 2 - 7 } & \multicolumn{3}{c}{ Total $\mathrm{n}=251$} & \multicolumn{7}{c}{ Com neuropatia $\mathrm{n}=41$} & Sem neuropatia $\mathrm{n}=41$ & $\mathrm{p}$ \\
\cline { 2 - 7 } & $\mathrm{n}$ & $\%$ & $\mathrm{n}$ & $\%$ & $\mathrm{n}$ & $\%$ & $\mathrm{p}$ \\
\cline { 2 - 7 } PSP $^{*}$ alterado & 75 & 29,9 & 21 & 51,2 & 54 & 25,7 & $\mathbf{0 , 0 0 1}$ \\
Descritores de dor & & & & & & & \\
Queimação & 80 & 31,9 & 19 & 46,3 & 61 & 29 & $\mathbf{0 , 0 0 4}$ \\
Dormência & 62 & 24,7 & 15 & 36,6 & 47 & 22,4 & 0,109 \\
Formigamento & 53 & 21,1 & 17 & 41,5 & 36 & 17,1 & $\mathbf{0 , 0 0 2}$ \\
Fadiga & 112 & 44,6 & 15 & 36,6 & 97 & 46,2 & 0,082 \\
Câimbras & 79 & 31,5 & 15 & 36,6 & 64 & 30,5 & 0,394 \\
Prurido & 24 & 9,6 & 7 & 17,1 & 17 & 8,1 & 0,115 \\
Alfinetada e/ou agulhada & 64 & 25,5 & 17 & 41,5 & 47 & 22,4 & $\mathbf{0 , 0 3}$ \\
Outro & 7 & 2,8 & 1 & 2,4 & 6 & 2,9 & 0,412 \\
\hline
\end{tabular}

${ }^{*}$ PSP- perda da sensibilidade protetora plantar

Referente à avaliação da QV, o índice geral de QV pelo instrumento SF6D obteve média de 0,78 , de maneira que os pacientes com neuropatia tiveram menor escore, porém sem haver diferença entre os grupos $(\mathrm{p}>0,05)$. Observou-se que, no geral, o domínio dor foi o mais afetado 
entre os participantes, seguido de vitalidade e saúde mental. O domínio menos afetado foi o aspecto social, sem ocorrer, contudo, diferença entre os grupos de estudo (p>0,05) (Tabela 4).

Tabela 4 - Comparação dos domínios de qualidade de vida alterados de indivíduos com DM tipo 2 com e sem neuropatia ( $\mathrm{n}=251$ ), Região Oeste, Brasília-DF, 2017.

\begin{tabular}{|c|c|c|c|c|c|c|c|}
\hline & \multicolumn{6}{|c|}{ Escala de dor LANSS } & \multirow{3}{*}{$\mathrm{p}$} \\
\hline & \multicolumn{2}{|c|}{ Total $n=251$} & \multicolumn{2}{|c|}{ Com neuropatia $n=41$} & \multicolumn{2}{|c|}{ Sem neuropatia $n=210$} & \\
\hline & $\mathrm{N}$ & $\%$ & $\mathrm{~N}$ & $\%$ & $\mathrm{~N}$ & $\%$ & \\
\hline Capacidade funcional & 161 & 64,1 & 31 & 75,6 & 130 & 61,9 & 0,094 \\
\hline Limitação global & 152 & 60,5 & 27 & 65,9 & 125 & 59,5 & 0,448 \\
\hline Aspectos sociais & 124 & 49,4 & 22 & 53,7 & 102 & 48,6 & 0,551 \\
\hline Dor & 229 & 91,2 & 40 & 97,6 & 189 & 90,0 & 0,117 \\
\hline Saúde mental & 187 & 74,5 & 35 & 85,4 & 152 & 72,4 & 0,081 \\
\hline Vitalidade & 200 & 79,6 & 33 & 80,5 & 167 & 80,0 & 0,881 \\
\hline \multicolumn{8}{|l|}{ EGQV-SF6D* } \\
\hline$\left({ }^{\dagger} \mathrm{M} \pm \mathrm{DP}^{\ddagger}\right)$ & \multicolumn{2}{|c|}{$0,78 \pm 0,09$} & $0,77 \pm 0,06$ & & \multicolumn{2}{|l|}{$0,79 \pm 0,10$} & 0,090 \\
\hline
\end{tabular}

* Escore Geral de Qualidade de Vida SF6D (EGQV-SF6D): ' Média (M), " Desvio Padrão (DP)

\section{Discussão}

Em relação ao perfil sociodemográfico, a prevalência do sexo feminino nesta pesquisa é um dado observado em outros estudos realizados entre indivíduos com DM.,11 A maioria dos pacientes eram casados e encontravam-se na faixa etária de 51 a 60 anos. Sabe-se que a presença do companheiro/cônjuge influencia na dinâmica familiar e no processo de autocuidado do paciente com DM, o que pode contribuir nesse processo de ajuda., ${ }^{3,5}$ Outros estudos com a mesma população demonstraram perfis semelhantes em relação à idade e ao estado civil. . $^{7,10}$

No presente estudo, houve ainda prevalência de idosos com baixa escolaridade e renda, o que reflete a carência socioeconômica da população pesquisada. Ainda assim, resultados de algumas pesquisas corroboram esses dados. ${ }^{11-12} \mathrm{O}$ nível de escolaridade quando relacionado à saúde é um fator relevante, já que indivíduos com poucos anos de estudo possuem maior tendência a compreenderem menos a doença e o tratamento, bem como as consequências do controle inadequado. ${ }^{6,11}$ 
O DM2 geralmente está associado a algum tipo de comorbidade, sendo a Hipertensão Arterial Sistêmica (HAS) uma das mais frequentes, fato observado neste estudo. ${ }^{4}$ Deve-se atentar a isso, pois a associação entre o DM2 e a HAS agrega maiores riscos no que diz respeito ao desenvolvimento de complicações crônicas do DM2, além de aumentar a morbimortalidade por doenças cardiovasculares. ${ }^{2}$

No entanto, além da HAS, outras condições também são fatores de risco para o desenvolvimento da ND, bem como idade avançada, hiperglicemia constante, hipertrigliceridemia, gênero masculino, maior tempo de DM, hemoglobina glicosilada (HbA1C) maior que 7\%, insulinoterapia, dislipidemia, albuminúria e obesidade..$^{2,4,12}$ Esses fatores mantêm o ciclo de estresse oxidativo, promovem sinais inflamatórios, causam danos celulares e resultam na aceleração do desenvolvimento da ND. ${ }^{10}$

Conforme exposto anteriormente, o inadequado controle glicêmico é um dos fatores desencadeantes do desenvolvimento de ND. A hiperglicemia diminui a capacidade de eliminação dos radicais livres, causando comprometimento do metabolismo neuronal e disfunção das fibras nervosas, o que consequentemente diminui a velocidade de condução. ${ }^{2,10}$ Autores afirmam que o controle metabólico eficaz reduz o risco de complicações. ${ }^{11} \mathrm{E}$, mesmo a curto prazo, este controle pode melhorar tanto as alterações metabólicas em glicose e lipídeos quanto a sensibilidade vibratória referida por esses pacientes. ${ }^{12-13}$

O maior tempo de diagnóstico de DM é outro fator que aumenta os riscos para o desenvolvimento de complicações. Neste estudo prevaleceram indivíduos com DM, com tempo de diagnóstico de até 10 anos. Outras pesquisas tiveram resultados próximos, variando de 9 a 14 anos. ${ }^{11,20-21}$ No entanto, algumas investigações apresentaram achados superiores com tempo médio maior que 15 anos. ${ }^{12-13,17}$ Pesquisadores afirmam ainda que a ND ocorre, principalmente, após os 10 primeiros anos de convívio com a doença. ${ }^{2,13,18}$

Dessa forma, é importante a equipe de enfermagem trabalhar a educação em saúde frente ao controle glicêmico, devido à existência de evidências segundo as quais, durante o pré- 
Comparação da dor e qualidade de vida entre indivíduos com e sem neuropatia diabética | 12

diabetes, já pode ocorrer a ND. ${ }^{6} \mathrm{O}$ enfermeiro deve incentivar o controle glicêmico persistente com vistas a prevenir e/ou retardar a complicação do DM2.

Os resultados deste estudo demonstraram uma prevalência de ND semelhante aos encontrados por outros autores. ${ }^{19-21}$ Em pesquisas internacionais na América do Norte, a prevalência de ND foi aproximada com a do presente estudo. ${ }^{12-13} \mathrm{Na}$ África entre 252 pacientes com DM avaliados, a prevalência de ND foi de $18,0 \% .{ }^{22}$ Em uma investigação desenvolvida no Brasil, foi identificada uma prevalência de ND de $34,1 \% .{ }^{17}$ Contudo, em indivíduos com DM diagnosticados, há mais de 10 anos esse número pode ser maior. ${ }^{1-2,5,12-13,17,23}$

Uma questão considerável diante da ND é a dificuldade do diagnóstico precoce. É comum que, ao descobrir o DM, o paciente já tenha a doença em estágio avançado, muitas vezes com complicações ou até mesmo com a própria ND. Em razão disso, a investigação precoce do DM é essencial visando a protelar outros problemas associados. ${ }^{2}$ Uma vez conhecido que o paciente tem DM, o enfermeiro tem papel indispensável no sucesso do tratamento e na prevenção de complicações. O Ministério da Saúde recomenda que o enfermeiro deva realizar consultas de enfermagem, uma vez que a ND pode ser dolorosa e não dolorosa. ${ }^{4}$

Quando o paciente evolui com ND sem a presença de dor, pode ter como consequência grave a insensibilidade dos pés, formação de úlcera do pé diabético (UPD), amputação e mortalidade precoce. O enfermeiro deve reconhecer os indivíduos de risco, realizar avaliação dos pés e orientar cuidados objetivando prevenir e rastrear precocemente casos de ND.,17,24 Estas medidas possibilitam que os atendimentos realizados na atenção primária devem compreender a avaliação da PSP, característica do desenvolvimento da neuropatia, a importância do autocuidado e do cuidado diário com os pés. ${ }^{2,4}$

Quanto ao rastreamento da ND por meio da avaliação dos pés, a Sociedade Brasileira de Diabetes recomenda pesquisar a PSP plantar por meio do teste de monofilamento $10 \mathrm{~g}$ e dos testes sensitivos. Quando ocorre insensibilidade ao monofilamento e um ou mais testes 
sensitivos motores (sensibilidade vibratória, dolorosa e reflexo aquileu), está presente a PSP. O monofilamento de Semmes-Weinstein tem o uso recomendado por demonstrar alta especificidade no diagnóstico de ND, além da facilidade de utilização e relação custobenefício. ${ }^{2,24}$ Em especial, na avaliação da dor neuropática pela escala LANSS, é utilizado o palito e na PSP também se utilizam, na avaliação da sensibilidade dolorosa, medidas de avaliação e caracterização da dor neuropática., ${ }^{2,4}$

Nesse sentido, em virtude de, na atenção básica, não haver equipamentos confirmatórios de neurocondução e da alta especificidade e relação custo-benefício, esta pesquisa fez o rastreamento do possível diagnóstico da neuropatia por meio da avaliação da sensibilidade tátil (monofilamento de $10 \mathrm{~g}$ ) e da escala de dor de LANSS, que reconhece se os mecanismos neuropáticos contribuem com a dor referida., ${ }^{2,46}$ Quando os exames eletrofisiológicos como eletroneuromiografia e potenciais evocados são utilizados para o diagnóstico, verifica-se o acometimento precoce do sistema nervoso, que pode chegar próximo de $100 \% .{ }^{2,5}$

Neste estudo, na avaliação do monofilamento, um terço dos participantes teve o teste alterado. A ND acomete as fibras nervosas do sistema nervoso periférico, especialmente dos MMII. ${ }^{9}$ O comprometimento é difuso, simétrico e distal e clinicamente, quando sintomática, cursa com sintomas sensitivos positivos, ou seja, apresenta resposta excessiva a um estímulo ou sensações espontâneas, como parestesia, queimação, formigamento e dor. ${ }^{16,20} \mathrm{Ou}$ ainda, sintomas sensitivos negativos, isto é, resposta reduzida a determinado estímulo, por exemplo, dormência e perda de sensibilidade nos membros acometidos. ${ }^{2,10}$ A particularidade marcante desses sintomas é o relato de exacerbação noturna. ${ }^{4}$ Tal fato foi observado neste estudo, pois frequentemente levantavam à noite devido à dor. Os resultados da pesquisa desenvolvida em Teresina foram semelhantes. ${ }^{25}$

Neste estudo, quase a totalidade dos pacientes referiram dor, sendo a maioria com presença de dor em pés ou panturrilhas. Alguns pesquisadores asseguram que um a cada cinco indivíduos com DM têm dor neuropática., ${ }^{2,5}$ Outros afirmam que 13 a $21 \%$ dos pacientes 
Comparação da dor e qualidade de vida entre indivíduos com e sem neuropatia diabética | 14

neuropáticos têm dor. ${ }^{26-27}$ Investigação como esta confirmou essas alegações e apresentou frequência de ND entre 16,7 e 75,0\% dos pacientes avaliados. ${ }^{7,23}$

Os principais descritores da dor foram fadiga, queimação, câimbra e dormência. Especialistas descrevem a dor neuropática em indivíduos com DM como picada, ardência intensa, choque, queimação, fadiga, dor em pontada, lancinante, parestesia, dormência e câimbras. ${ }^{5,10}$ Em outros estudos para avaliação da neuropatia dolorosa, os descritores mais comuns são queimação, formigamento ou dormência. ${ }^{19,25-27}$ Além disso, alguns pacientes também descrevem desequilíbrio, quedas, choques, picadas, agulhadas, bem como desconforto ou dor ao toque de lençóis e cobertores e piora em repouso. ${ }^{5}$

Nesta investigação, a dor moderada e intensa foi prevalente entre os grupos. Em uma pesquisa realizada na Espanha com objetivo de analisar a ND e ND dolorosa de 130 indivíduos, verificou-se que 50,0\% dos neuropáticos referiram dor moderada. ${ }^{27}$ Por outro lado, a ND também pode se desenvolver de forma assintomática devido à perda total de sensibilidade. ${ }^{24,28-29}$ Essa perda de sensibilidade somada à não presença de dor deve ser considerada com atenção pela equipe de enfermagem visto que são pacientes de risco de lesões de MMII.

Ressalta-se que os pacientes com ND dolorosa convivem com dor de forma intensa e se torna crônica trazendo prejuízos ao seu cotidiano e ao seu humor. ${ }^{2}$ A avaliação da dor deve fazer parte da rotina dos enfermeiros, tanto na investigação da intensidade e da duração, quanto principalmente na qualidade que descreve a dor da ND (queimação, fadiga e dormência). ${ }^{11,16}$ Esses descritores são de origem neuropática e evidenciam a responsabilidade do enfermeiro em traçar plano de cuidados para o controle da dor e para a redução dos impactos físicos e emocionais na vida dos pacientes com DM2. Salienta-se que a dor crônica necessita de terapêutica medicamentosa e atuação entre equipe interdisciplinar (médico, enfermeiro e farmacêutico), especialmente na atenção básica. 
15 | Silva ACG, Stival MM, Funghetto SS, Volpe CRG, Funez MI, LimaVI LR

Somado a sintomas dolorosos, pode ocorrer alta incidência de quedas, depressão, limitações, incapacidade funcional e diminuição da sobrevida. A Sociedade Brasileira de Diabetes considera a neuropatia um problema de saúde pública que afeta diretamente a QV dos pacientes. $^{2}$ Autores revelam que a maioria dos casos possuidores de sequelas relacionadas a complicações crônicas do DM apresentam QV inferior, quando comparados com os que não são acometidos por complicações. ${ }^{5,22,25}$

Nesta pesquisa a média do escore de QV, por meio do SF6D, foi semelhante à encontrada por outro estudo recente (média $=0,85) .{ }^{8}$ Autores afirmam a utilidade desse instrumento na avaliação da QV na atenção básica ${ }^{7}$ por ser rápido, fácil, de confiabilidade e reprodutibilidade certificada.,14,16

Em outra investigação com 756 indivíduos com DM, a dimensão de QV mais prejudicada pelo questionário SF12D foi a de estado geral de saúde, estado físico e saúde mental. ${ }^{26}$ Uma pesquisa conduzida em Montes Claros com 201 idosos teve o objetivo de avaliar o impacto do tempo de diagnóstico do DM sobre a QV de idosos atendidos em UBS, por meio do SF-36. Os resultados demonstraram que o tempo de DM superior a 10 anos influenciou negativamente a QV de idosos, principalmente na capacidade funcional, na vitalidade e na saúde mental. O aumento da intensidade da dor e maior limitação física e emocional dos idosos podem ser considerados os fatores de maior influência na redução da QV. ${ }^{30}$

Neste estudo, os domínios mais afetados foram dor, vitalidade, saúde mental e capacidade funcional. Ainda em concordância com estes achados, outra investigação realizada em Porto Alegre com 211 pacientes da atenção básica evidenciou que os domínios dor e vitalidade contribuem significativamente para redução da QV na população. ${ }^{8}$ Já, no Canadá, uma pesquisa identificou que o DM acarreta prejuízos na QV, sobretudo nos domínios físico e mental. ${ }^{29}$

Devido aos sintomas de dor, a ND dolorosa tem repercussão negativa na QV de indivíduos com DM relacionada com alterações causadas nas atividades recreativas e sociais, e também nas questões relacionadas com trabalho e mobilidade. Além disso, o tratamento da dor 
Comparação da dor e qualidade de vida entre indivíduos com e sem neuropatia diabética | 16

requer o uso de medicamentos e cuidados complementares que demandam recursos financeiros, sociais e educacionais peculiares, muitas vezes, inacessíveis. ${ }^{2,4,12}$

Ressalta-se ainda que, além de prejuízos no domínio dor, os participantes desta pesquisa também tiveram prejuízos nos outros domínios de QV. Portanto, é evidente que os impactos negativos gerados pelo DM2 não se restringem somente à neuropatia, mas também a mudanças no estilo de vida devido à terapêutica, efeitos colaterais das medicações, preocupações com a cronicidade da doença, custos com questões relativas à doença, além do apoio social e familiar. ${ }^{1,23,29}$

Acredita-se que os resultados desta pesquisa trazem contribuições relevantes para a enfermagem, pois podem favorecer a assistência na atenção primária, incentivando a utilização de escalas padronizadas, como caracterização de dor e escala de LANSS, que, de forma rápida, podem identificar pacientes que tenham dor com características de ND. Estes resultados também podem direcionar e melhorar a avaliação de enfermagem em pessoas que vivem com condições crônicas como o DM.

A limitação deste estudo foi o desenho transversal, o qual não permite o estabelecimento de relações de causa e efeito. Além disso, as variáveis como colesterol, triglicerídeos, hemoglobina glicada e glicemia não foram investigadas.

\section{Conclusão}

O estudo demonstrou que a maioria dos pacientes com neuropatia apresenta dor intensa, o que os leva a acordar à noite devido a essa dor. A média de intensidade de dor foi significativamente maior no grupo com neuropatia. Os principais domínios de QV afetados dos pacientes com neuropatia foram: dor, vitalidade, saúde mental e capacidade funcional. Observou-se que o perfil dos pacientes não neuropáticos é semelhante ao dos neuropáticos, porém aqueles com neuropatia referem dor do tipo queimação, formigamento e alfinetada e/ou agulhada. 
Posto isso, são necessários cuidados que visam à prevenção da ND e que promovam a QV desses pacientes, como rastreamento precoce da dor com características de ND, somada à avaliação dos pés, modificações de hábitos de vida, alterações de padrões alimentares, controle glicêmico adequado, prática de atividade física, controle do DM2 e acompanhamento contínuo pela equipe de enfermagem na atenção básica.

\section{Referências}

1. International Diabetes Federation (IDF). IDF Diabetes Atlas. 9th edition 2019 [Internet]. Brussels: International Diabetes Federation; 2019 [cited 2020 Dec 12]. Available from: http://www.diabetesatlas.org/ 2. Sociedade Brasileira de Diabetes (SBD). Diretrizes da Sociedade Brasileira de Diabetes (2019-2020) [Internet]. São Paulo: Clammad; 2019 [acesso em 2020 dez 12]. Disponível em: https://www.diabetes.org.br/profissionais/images/DIRETRIZES-COMPLETA-2019-2020.pdf

3. Côrrea K, Gouvêa GR, Silva MAV, Possobon RF, Barbosa LFLN, Pereira AC, et al. Qualidade de vida e característica de pacientes diabéticos. Ciênc Saúde Colet. 2017;22(3):921-30. doi: 10.1590/141381232017223.24452015

4. Ministério da Saúde (BR). Manual do pé diabético: estratégias para o cuidado da pessoa com doença crônica [Internet]. Brasília (DF): Ministério da Saúde; 2016 [acesso em 2020 dez 12]. Disponível em: http://189.28.128.100/dab/docs/portaldab/publicacoes/manual_do_pe_diabetico.pdf

5. Shillo P, Sloan G, Greig M, Hunt L, Selvarajah D, Elliott J, et al. Painless and painless diabetic neuropathies: what's the difference? Curr Diab Rep. 2019;19(6):27-32. doi: 10.1007/s11892-019-1150-5

6. Grisold A, Callaghan BC, Feldman EL. Mediators of diabetic neuropathy: is hyperglycemia the only culprit? Curr Opin Endocrinol Diabetes Obes. 2017;24(2):103-11. doi: 10.1097/MED.0000000000000320

7. Jiao F, Wong CKH, Gangwani R, Tan KCB, Tang SCW, Lam CLK. Health-related quality of life and health preference of Chinese patients with diabetes mellitus managed in primary care and secondary care setting: decrements associated with individual complication and number of complications. Health Qual Life Outcomes. 2017;15(1):125. doi: 10.1186/s12955-017-0699-4

8. Berwanger DD, Filippin LI, Saldanha RP. SF-6D como uma medida rápida de qualidade de vida: um estudo descritivo na atenção básica de saúde. Rev Inspirar [Internet]. 2016 [acesso em 2020 dez 12];11(4):20-6. Disponível em: https://www.inspirar.com.br/wp-content/uploads/2016/12/artigo3-sf-6d-.pdf

9. Silva REG, Morais ACS, Godoi CD, Garcia OAG, Rabelo Neto WN. Avaliação sensório-motora e sua correlação com qualidade de vida em portadores de diabetes mellitus. South Am J Basic Educ Tech Technol [Internet]. 2017 [cited 2020 Dec 12];4(1):118-32. Available from: https://periodicos.ufac.br/index.php/SAJEBTT/article/view/1140 
Comparação da dor e qualidade de vida entre indivíduos com e sem neuropatia diabética | 18

10. Sociedade Brasileira de Diabetes (SBD). Neuropatia diabética: posicionamento da Associação Americana de Diabetes [Internet]. São Paulo: Sociedade Brasileira de Diabetes; 2019 [acesso em 2020 dez 12]; Disponível em: https:/www.diabetes.org.br/profissionais/images/2019/posicionamento2019/Posicionamento-NeuropatiaDiabetica-SBD.pdf

11. Lima LR, Stival MM, Funghetto SS, Volpe CRG, Rehem TCMSB, Santos WS, et al. Lower quality of life, lower limb pain with neuropathic characteristics, female sex, and ineffective metabolic control are predictors of depressive symptoms in patients with type 2 diabetes mellitus treated in primary care. Int J Diabetes Develop Ctries. 2018;39(6):463-70. doi: 10.1007/s13410-018-0667-5

12. Liu X, Xu Y, An M, Zeng Q. The risk factors for diabetic peripheral neuropathy: a meta-analysis. PLoS One. 2019 Feb 20;14(2):e0212574. doi: 10.1371/journal.pone.0212574

13. Girach A, Julian TH, Varrassi G, Paladini A, Vadalouka A, Zis P. Quality of life in painful peripheral neuropathies: a systematic review. Pain Res Manag. 2019:2091960. doi: 10.1155/2019/2091960

14. Schestatsky P, Félix-Torres V, Chaves MLF, Câmara-Ehlers B, Mucenic T, Caumo W, et al. Brazilian Portuguese validation of the leeds assessment of neuropathic symptoms and signs for patients with chronic pain. Pain Med. 2011 Oct;12(10):1544-50. doi:10.1111/j.1526-4637.2011.01221.x

15. Pimenta CAM, Teixeira MJ. Questionário de dor McGill: proposta de adaptação para a língua portuguesa. Rev Esc Enferm USP. 1996;30(3):473-83. doi: 10.1590/S0080-62341996000300009

16. Campolina AG, Bortoluzzo AB, Ferraz MB, Ciconelli RM. Validação da versão brasileira do questionário genérico de qualidade de vida short-form 6 dimensions (SF-6D Brasil). Ciênc Saúde Colet. 2011;16(7):3103-10. https://doi.org/10.1590/S1413-81232011000800010

17. Aguiar FLXS, Ramos LFP, Bichara CNC. Detecção de dor com características neuropáticas em pacientes com diabetes mellitus atendidos na atenção básica. BrJP. 2018;1(1):15-20. doi: 10.5935/2595-0118.20180005

18. Isomura T, Sumitani M, Matsudaira K, Kawaguchi M, Inoue R, Hozumi J, et al. Development of the Japanese version of the leeds assessment of the neuropathic symptoms and signs pain scale: diagnostic utility in a clinical setting. Pain Pract. 2017;17(6):800-7. doi: 10.1111/papr.12528

19. Say B, Ergün U, Yıldız A, Alpua M, Arıkan Durmaz Ş, Turgal E. Detection of pain severity with the full cup test in painful diabetic peripheral neuropathy. Agri. 2020 Apr;32(2):85-90. doi: 10.14744 agri.2019.82653

20. Cevik AB, Olgun N. The predictors of painful diabetic neuropathy and its effect on quality of life. Pain Manag Nurs. 2021;S1524-9042(21):00126-0. doi: 10.1016/j.pmn.2021.04.002

21. Ponirakis G, Elhadd T, Chinnaiyan S, Dabbous Z, Siddiqui M, Al-Muhannadi H, et al. Prevalence and risk factors for painful diabetic neuropathy in secondary healthcare in Qatar. J Diabetes Investig. 2019;10(6):1558-64. doi: 10.1111/jdi.13037

22. Maiga Y, Diallo S, Konipo FDN, Sangho O, Sangaré M, Diallo SH, et al. Diabetic polyneuropathy with/out neuropathic pain in Mali: a cross-sectional study in two reference diabetes treatment centers in Bamako (Mali), West Africa. PLoS One. 2020;15(11):e0241387. doi: 10.1371/journal.pone.0241387 
23. Dutra LMA, Moura MC, Prado FA, Lima GO, Melo MC, Fernandez RNM, et al. Is it possible to substitute the monofilament test for the Ipswich Touch Test in screening for peripheral diabetic neuropathy? Diabetol Metab Syndr. 2020;12(27):1-6. doi: 10.1186/s13098-020-00534-2

24. Feitosa TF, Dantas MQS, Silva CB, Pereira A. Monofilament for preventing the diabetic foot: an integrative review of the literature. Online Braz J Nurs. 2016;15(2):291-301. doi: 10.17665/1676-4285.20165277

25. Naranjo C, Ortega-Jiménez P, Del Reguero L, Moratalla G, Failde I. Relationship between diabetic neuropathic pain and comorbidity. Their impact on pain intensity, diabetes complications and quality of life in patients with type-2 diabetes mellitus. Diabetes Res Clin Pract. 2020;165(6):e108236. doi: 10.1016/j.diabres.2020.108236

26. Silveira IA, Oliveira BGRB, Oliveira AP, Andrade NC. Padrão da dor de pacientes com úlcera de pernas. Rev Enferm UFPE On Line [Internet]. 2017 [cited 2020 Dec 12];11(2):617-24. Available from: https://periodicos.ufpe.br/revistas/revistaenfermagem/article/view/11981/14537

27. Pop-Busui R, Boulton AJ, Feldman EL, Bril V, Freeman R, Malik RA, et al. Diabetic neuropathy: a position statement by the American Diabetes Association. Diabetes Care. 2017;40(1):136-54. doi: $10.2337 / \mathrm{dc} 16-2042$

28. Lima LR, Stival MM, Funghetto SS, Silva ICR, Rehem TCMSB, Santos WS, et al. Neuropatia e dor nos membros inferiores: sinais percussores do pé diabético. In: Parisi MCR, Leite CR, Rosa MFF. Interdisciplinaridade no contexto das doenças dos pés no diabetes: tratamento clínicos, políticas públicas e tecnologias em saúde. $1^{\mathrm{a}}$ ed. Mossoró: EDUERN. 2021.

29. Feldman EL, Callaghan BC, Pop-Busui R, Zochodne DW, Wright DE, Bennett DL, et al. Diabetic neuropathy. Nat Rev Dis Primers. 2019 Jun 13;5(1):42. doi: 10.1038/s41572-019-0092-1

30. Matias COF, Matias COF, Alencar BA. Qualidade de vida em idosos portadores de Diabetes Mellitus Tipo 2 atendidos em Unidades Básicas de Saúde de Montes Claros/MG. Rev Bras Qual Vida. 2016;8(2):119-29. doi: 10.3895/rbqv.v8n2.3841

Editora Científica: Tânia Solange Bosi de Souza Magnago

Editora associada: Alexa Pupiara Flores Coelho

Fomento / Agradecimento: Fundação de Amparo e pesquisa do Distrito Federal-FAP/DF, a aluna de iniciação científica Ananda Gonçalves Menezes que ajudou a concluir esta pesquisa e integrantes do Grupo de Pesquisa Saúde, Cuidado e Envelhecimento- GEPSEN/UNB.

\section{Autor correspondente}

Luciano Ramos de Lima

E-mail: ramosll@unb.br

Endreço: Universidade de Brasília-Campus Ceilândia/Curso de enfermagem, Centro Metropolitano, lote 01, Ceilândia Sul, Sala A1-28/15, Brasília-DF, Brasil. 
Comparação da dor e qualidade de vida entre indivíduos com e sem neuropatia diabética | 20

CEP: $72220-140$

\section{Contribuições de Autoria}

\section{1 - Ana Catarina Guimarães Silva}

Concepção ou desenho do estudo/pesquisa e análise e/ou interpretação dos dados,

\section{2 - Marina Morato Stival}

Concepção ou desenho do estudo/pesquisa; análise e/ou interpretação dos dados, revisão final com participação crítica e intelectual no manuscrito.

\section{3 - Silvana Schwerz Funghetto}

Concepção ou desenho do estudo/pesquisa

\section{4 - Cris Renata Grou Volpe}

Análise e/ou interpretação dos dados,

\section{5 - Mani Indiana Funez}

Concepção ou desenho do estudo/pesquisa; análise e/ou interpretação dos dados, revisão final com participação crítica e intelectual no manuscrito.

\section{6 - Luciano Ramos de Lima}

Concepção ou desenho do estudo/pesquisa; análise e/ou interpretação dos dados, revisão final com participação crítica e intelectual no manuscrito.

\section{Como citar este Artigo}

Comparison of pain and quality of life between individuals with and without diabetic neuropathy. Silva ACG, Stival MM, Funghetto SS, Volpe CRG, Funez MI, LimaVI LR. Rev. Enferm. UFSM. 2021 [Access in: Year Month Day]; vol.11 e62: 1-120. DOI: https://doi.org/10.5902/2179769263722 